\title{
Quantitative multiparametric MRI predicts response to neoadjuvant therapy in the community setting
}

John Virostko ${ }^{1,2,3,4+}$ 일 Anna G. Sorace ${ }^{5,6,7+}$, Kalina P. Slavkova ${ }^{8}$, Anum S. Kazerouni ${ }^{9}$, Angela M. Jarrett ${ }^{4}$, Julie C. DiCarlo ${ }^{2,4}$, Stefanie Woodard ${ }^{5}$, Sarah Avery ${ }^{10}$, Boone Goodgame ${ }^{11}$, Debra Patt ${ }^{12}$ and Thomas E. Yankeelov 1,2,3,4,13,14*

\begin{abstract}
Background: The purpose of this study was to determine whether advanced quantitative magnetic resonance imaging (MRI) can be deployed outside of large, research-oriented academic hospitals and into community care settings to predict eventual pathological complete response ( $P C R$ ) to neoadjuvant therapy (NAT) in patients with locally advanced breast cancer.

Methods: Patients with stage II/III breast cancer $(N=28)$ were enrolled in a multicenter study performed in community radiology settings. Dynamic contrast-enhanced (DCE) and diffusion-weighted (DW)-MRI data were acquired at four time points during the course of NAT. Estimates of the vascular perfusion and permeability, as assessed by the volume transfer rate $\left(K^{\text {trans }}\right)$ using the Patlak model, were generated from the DCE-MRI data while estimates of cell density, as assessed by the apparent diffusion coefficient (ADC), were calculated from DW-MRI data. Tumor volume was calculated using semi-automatic segmentation and combined with $K^{\text {trans }}$ and ADC to yield bulk tumor blood flow and cellularity, respectively. The percent change in quantitative parameters at each MRI scan was calculated and compared to pathological response at the time of surgery. The predictive accuracy of each MRI parameter at different time points was quantified using receiver operating characteristic curves.

Results: Tumor size and quantitative MRI parameters were similar at baseline between groups that achieved pCR $(n=8)$ and those that did not $(n=20)$. Patients achieving a pCR had a larger decline in volume and cellularity than those who did not achieve pCR after one cycle of NAT $(p<0.05)$. At the third and fourth MRI, changes in tumor volume, $K^{\text {krans }}, A D C$, cellularity, and bulk tumor flow from baseline (pre-treatment) were all significantly greater $(p<0.05)$ in the cohort who achieved pCR compared to those patients with non-pCR.

Conclusions: Quantitative analysis of DCE-MRI and DW-MRI can be implemented in the community care setting to accurately predict the response of breast cancer to NAT. Dissemination of quantitative MRI into the community setting allows for the incorporation of these parameters into the standard of care and increases the number of clinical community sites able to participate in novel drug trials that require quantitative MRI.
\end{abstract}

Keywords: NAT, Diffusion, Dynamic contrast enhanced, DW-MRI, DCE-MRI

*Correspondence: thomas.yankeelov@utexas.edu

†John Virostko and Anna G. Sorace have contributed equally to this study

${ }^{1}$ Department of Diagnostic Medicine, University of Texas at Austin, Austin, TX 78712, USA

Full list of author information is available at the end of the article

\section{Introduction}

Quantitative imaging allows for the characterization of biological phenomena from radiological data and has developed to the point where it is regularly incorporated original author(s) and the source, provide a link to the Creative Commons licence, and indicate if changes were made. The images or other third party material in this article are included in the article's Creative Commons licence, unless indicated otherwise in a credit line to the material. If material is not included in the article's Creative Commons licence and your intended use is not permitted by statutory regulation or exceeds the permitted use, you will need to obtain permission directly from the copyright holder. To view a copy of this licence, visit http://creativecommons.org/licenses/by/4.0/. The Creative Commons Public Domain Dedication waiver (http://creativeco mmons.org/publicdomain/zero/1.0/) applies to the data made available in this article, unless otherwise stated in a credit line to the data. 
in oncology clinical trials in the academic setting $[1,2]$; however, it has yet to be expanded into the traditional radiology setting. With $85 \%$ of oncology patients receiving care and imaging from local or regional clinics [3], there is a need to move these advancements from academic institutes into community care facilities. Quantitative MRI has shown particular promise in predicting the response of breast tumors to neoadjuvant therapy (NAT) [4-6]. NAT, administration of therapy prior to definitive surgical resection of disease, is the standard-of-care approach for patients with locally advanced breast cancer (i.e., stage II-III). The main objectives of NAT are to improve overall patient survival by: (1) reducing the primary tumor burden for surgical resection, (2) treating clinically occult micrometastases, and (3) evaluating the impact of systemic therapies on breast cancer biology to improve selection of downstream therapeutic regimens [7-13]. Patients who achieve a pathological complete response (pCR, i.e., absence of viable tumor cells in the primary or local lymph nodes) following NAT have shown improved outcomes with increased survival rates $[7,8]$. Conversely, patients with residual disease (i.e., non$\mathrm{pCR}$ ) at the conclusion of NAT have an increased risk of early recurrence and poorer prognoses [14-18]. Thus, accurate and early assessment of response to NAT would provide the opportunity to replace an ineffective treatment with an alternative regimen, potentially improving outcomes while simultaneously avoiding or decreasing side effects of ineffective therapies. As quantitative MRI has been shown to predict the response of breast tumors early in the course of NAT in academic settings [19, 20], a natural progression toward implementation into standard of care is to integrate such techniques into both multi-site and community-based settings. As the majority of cancer patients receive their care in the community setting [21], this will allow for improved care for a dramatically large percentage of breast cancer patients.

Evaluating early changes in cellularity and vascularity through quantitative MRI provides the opportunity to obtain serial three-dimensional biological characterization of the tumor at baseline and in response to systemic treatments. Diffusion-weighted (DW)-MRI and dynamic contrast-enhanced (DCE)-MRI have the potential to characterize spatial and temporal alterations in breast cancer cells and the tumor microenvironment prior to downstream effects of changes in tumor size. Tracking alterations in the apparent diffusion coefficient (ADC), extracted from DW-MRI, has been used to predict the response of breast tumors to therapy [22-24]. The ADC correlates with cellularity [25-27] and may be a better predictor of eventual response than measurements of tumor size [28]. DCE-MRI pharmacokinetic parameters present quantitative information relating to the changes in vascular delivery and extracellular space and have been demonstrated to be predictive of pCR in breast tumors undergoing NAT [19, 23, 29, 30]. DCE-MRI parameters such as $K^{\text {trans }}$ (the volume transfer rate, related to vascular permeability and perfusion) can provide information on tumor vascularity. These emerging metrics have also shown clinical promise at informing advanced mathematical models that further describe the underlying cellular and biological features of the tumor during therapy $[31,32]$. While biopsy samples are an integral component of breast cancer diagnosis and treatment guidance, imaging provides a powerful and complementary, noninvasive tool to probe the entire tumor microenvironment. Advanced measurements quantifying underlying tumor biology represent a comprehensive and personalized approach to monitor and predict response in cancer care.

To maximize the impact of quantitative imaging of locally advanced breast cancer during NAT, quantitative imaging must be implemented and validated in the community setting. Further, these methods must be robust enough to be utilized in many different breast cancer subtypes and across various sequencing, timing, and types of systemic therapy. We have previously shown that quantitative MRI metrics (ADC from DW-MRI and $T_{1}$ mapping required for DCE-MRI) can be extracted from imaging protocols deployed in the community setting on phantoms and normal subjects with high repeatability and reproducibility [33]. The present study seeks to employ these techniques in the community setting to prospectively predict the response of locally advanced breast cancer and disseminate quantitative MRI beyond academia and toward routine application in the standardof-care setting.

\section{Methods}

\section{Study population}

Women $(N=28)$ who were previously diagnosed with locally advanced breast cancer (stages II-III) and prescribed NAT were enrolled into this prospective clinical imaging study. The median age of patients was 44.5 years old (range of 25-74 years). See Table 1 for a summary of patient demographics and treatment regimens. Treatment was selected by the patient's oncologist prior to study enrollment. No hormonal therapy was performed in this population.

\section{General study design}

Patients underwent quantitative MRI at one of two community imaging clinics (separate and in addition to standard-of-care imaging). In this study, a 'communitycare imaging center' was defined as a non-academic, non-research setting (i.e., does not actively train any medical residents). Seven study participants received all 
Table 1 Clinical features of the study population (pathological complete response, pCR)

\begin{tabular}{|c|c|c|c|c|}
\hline Patient \# & Age [years] & ER/PR/HER2 & Therapeutic regimen & $\begin{array}{l}\text { Pathological } \\
\text { response }\end{array}$ \\
\hline 1 & 54 & $+/-/-$ & doxorubicin/cyclophosphamide $\rightarrow$ paclitaxel & Non-pCR \\
\hline 2 & 41 & $+/+/+$ & doxorubicin/cyclophosphamide $\rightarrow$ paclitaxel/Herceptin & $\mathrm{pCR}$ \\
\hline 3 & 74 & $-/-/-$ & doxorubicin/cyclophosphamide $\rightarrow$ paclitaxel & Non-pCR \\
\hline 4 & 25 & $-/-/-$ & doxorubicin/cyclophosphamide $\rightarrow$ paclitaxel & Non-pCR \\
\hline 5 & 26 & $-/-/+$ & doxorubicin/cyclophosphamide $\rightarrow$ paclitaxel /Herceptin & $\mathrm{pCR}$ \\
\hline 6 & 41 & $-/-/-$ & Carboplatin/paclitaxel $\rightarrow$ doxorubicin/cyclophosphamide & $\mathrm{pCR}$ \\
\hline 7 & 37 & $-/-/-$ & Carboplatin/paclitaxel $\rightarrow$ doxorubicin/cyclophosphamide & Non-pCR \\
\hline 8 & 41 & $+/-/-$ & doxorubicin/cyclophosphamide $\rightarrow$ paclitaxel & $\mathrm{pCR}$ \\
\hline 9 & 47 & $+/+/-$ & doxorubicin/cyclophosphamide $\rightarrow$ paclitaxel & $\mathrm{pCR}$ \\
\hline 10 & 54 & $+/+/-$ & doxorubicin/cyclophosphamide $\rightarrow$ paclitaxel & Non-pCR \\
\hline 11 & 59 & $-/-/-$ & Pembrolizumab (or placebo)/Carboplatin/paclitaxel & Non-pCR \\
\hline 12 & 63 & $+/+/-$ & doxorubicin/cyclophosphamide $\rightarrow$ paclitaxel & Non-pCR \\
\hline 13 & 27 & $+/+/-$ & doxorubicin/cyclophosphamide $\rightarrow$ paclitaxel & Non-pCR \\
\hline 14 & 32 & $+/+/+$ & Taxotere/Carboplatin/Herceptin/Pertuzumab & Non-pCR \\
\hline 15 & 52 & $-/-/-$ & Carboplatin/paclitaxel $\rightarrow$ doxorubicin/cyclophosphamide & Non-pCR \\
\hline 16 & 38 & $-/-/-$ & Carboplatin/paclitaxel $\rightarrow$ doxorubicin/cyclophosphamide & $\mathrm{pCR}$ \\
\hline 17 & 38 & $-/-1-$ & Pembrolizumab (or placebo)/Carboplatin/paclitaxel & $\mathrm{pCR}$ \\
\hline 18 & 62 & $-/-/-$ & doxorubicin/cyclophosphamide $\rightarrow$ paclitaxel & Non-pCR \\
\hline 19 & 38 & $+/+/+$ & Taxotere/Carboplatin/Herceptin/Pertuzumab & Non-pCR \\
\hline 20 & 42 & $+/+/-$ & doxorubicin/cyclophosphamide $\rightarrow$ paclitaxel & Non-pCR \\
\hline 21 & 53 & $+/+/-$ & doxorubicin/cyclophosphamide $\rightarrow$ paclitaxel & Non-pCR \\
\hline 22 & 58 & $-/-/-$ & Pembrolizumab (or placebo)/Carboplatin/paclitaxel & Non-pCR \\
\hline 23 & 48 & $+/+/-$ & doxorubicin/cyclophosphamide $\rightarrow$ paclitaxel & Non-pCR \\
\hline 24 & 50 & $+/+/+$ & Taxotere/Carboplatin/Herceptin/Pertuzumab & Non-pCR \\
\hline 25 & 64 & $+/+/-$ & doxorubicin/cyclophosphamide $\rightarrow$ paclitaxel & Non-pCR \\
\hline 26 & 40 & $+/-/+$ & Taxotere/Carboplatin/Herceptin/Pertuzumab & $\mathrm{pCR}$ \\
\hline 27 & 31 & $-/-/-$ & doxorubicin/cyclophosphamide $\rightarrow$ paclitaxel & Non-pCR \\
\hline 28 & 54 & $-/-/-$ & Talazoparib & Non-pCR \\
\hline
\end{tabular}

imaging at a community hospital (not affiliated with an academic medical center) and the other 21 participants received all imaging at an outpatient imaging center. In the clinical workflow at these sites, the MRI technologists were directly involved and responsible for positioning the patients and deploying the research imaging protocols. Study personnel designed and installed the imaging protocol. Patients received a baseline session prior to beginning NAT (MRI 1) and following one round of NAT (MRI 2). For patients whose NAT included a second therapeutic regimen, MRIs 3 and 4 were performed prior to and after the first round of the second therapeutic regimen, respectively (Fig. 1A). For patients who maintained the same regimen throughout NAT, MRIs 3 and 4 were acquired after two and three rounds of NAT, respectively (Fig. 1B). Of the patients who enrolled in the study, three dropped out prior to MRI 2 and an additional two dropped out prior to MRI 3. None of these study dropouts achieved pCR. Additionally, no tumor was visible at MRI 2 for one patient who achieved pCR, no tumor was visible at MRI 3 for two patients who achieved pCR, and no tumor was visible at MRI 4 for three patients who achieved $\mathrm{pCR}$. As there was no viable tumor on which to measure ADC or $K^{\text {trans }}$ these measures were not performed at time points where tumor was no longer detectable.

\section{Image acquisition}

MRI examinations were performed on a Siemens 3T Magnetom Skyra MR scanner (Siemens Medical Solutions USA, Malvern, PA) at both clinical community imaging facilities equipped with either an 8- or 16-channel receive double-breast coil (Sentinelle, Invivo, Gainesville, Florida). Image acquisition parameters are summarized in Table 2. DW-MRI was acquired using a monopolar single-shot spin-echo echo planar imaging (EPI) sequence with diagonal monopolar diffusionencoding gradients. Six acquisitions were averaged for 


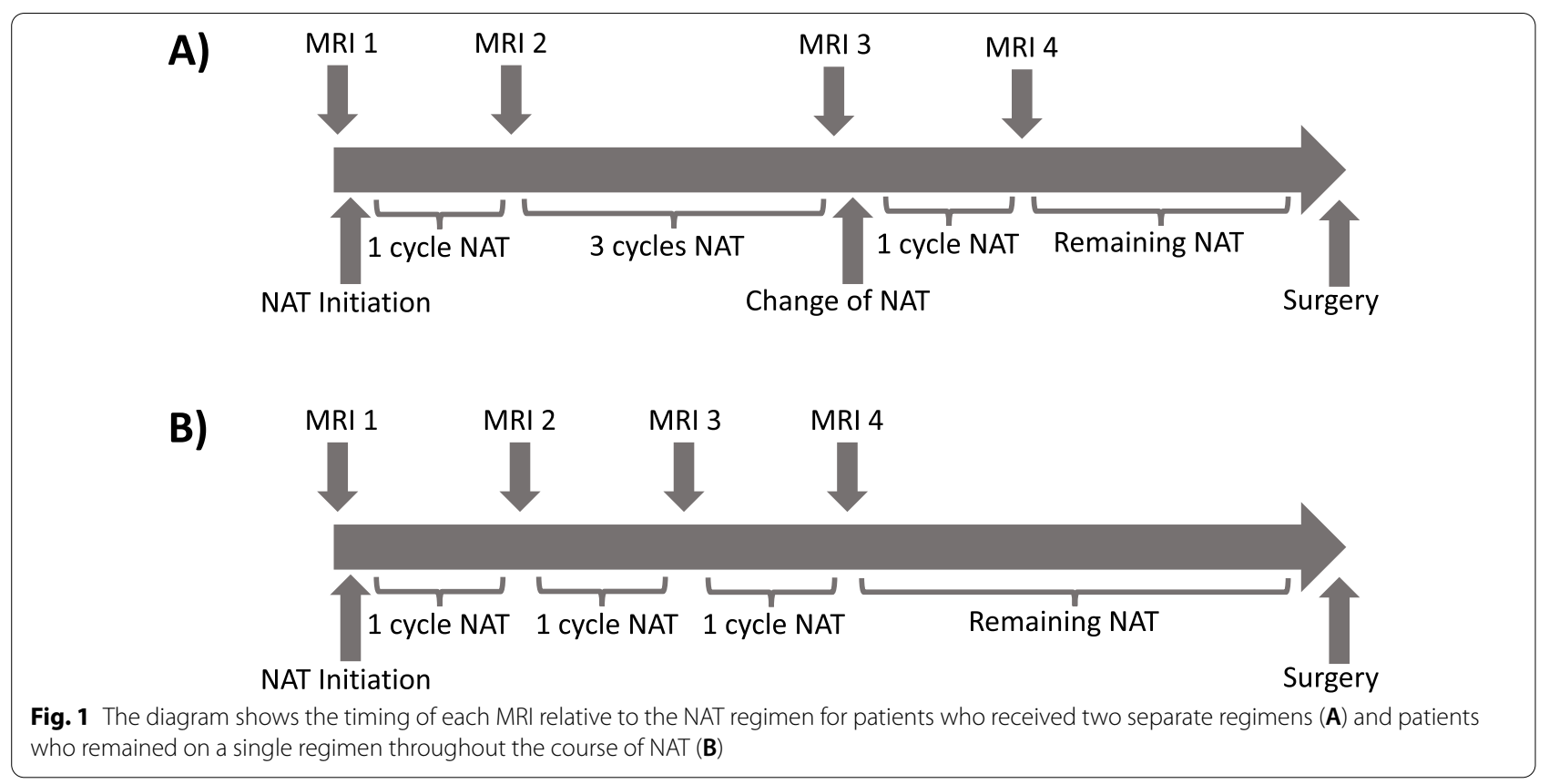

Table 2 MRI acquisition parameters (TR, repetition time; TE, echo time; FOV, field of view; GRAPPA, GeneRalized Autocalibrating Partial Parallel Acquisition)

\begin{tabular}{|c|c|c|c|c|c|}
\hline MRI parameters & Anatomical scan & DW-MRI & $B_{1}$ mapping & $\begin{array}{l}T_{1} \text {-mapping: variable flip } \\
\text { angle }\end{array}$ & DCE-MRI \\
\hline Scan sequence & $\begin{array}{l}T_{1} \text {-weighted 3D } \\
\text { gradient-echo } \\
\text { FLASH }\end{array}$ & $\begin{array}{l}\text { Single-shot spin-echo } \\
\text { (SE) echo planar (EPI) }\end{array}$ & 3D spoiled gradient echo & $\begin{array}{l}T_{1} \text {-weighted 3D spoiled } \\
\text { gradient echo }\end{array}$ & $\begin{array}{l}T_{1} \text {-weighted 3D } \\
\text { spoiled gradient } \\
\text { echo }\end{array}$ \\
\hline$T R(\mathrm{~ms})$ & 5.3 & 3000 & 8680 & 7.9 & 7.02 \\
\hline$T E(\mathrm{~ms})$ & 2.3 & 52 & 2 & 2.4 & 4.6 \\
\hline Flip angle $\left(^{\circ}\right)$ & 10 & 90 & 8 & $2,4,6,8,10,12,14,16,18,20$ & 6 \\
\hline Acquisition matrix & $256 \times 256$ & $128 \times 128$ & $96 \times 96$ & $192 \times 192$ & $192 \times 192$ \\
\hline FOV $(\mathrm{mm})$ & $256 \times 256$ & $256 \times 256$ & $256 \times 256$ & $256 \times 256$ & $256 \times 256$ \\
\hline Slice thickness (mm) & 1 & 5 & 5 & 5 & 5 \\
\hline GRAPPA acceleration factor & 2 & 2 & $\mathrm{~N} / \mathrm{A}$ & 3 & N/A \\
\hline Fat suppression & SPAIR & SPAIR & $\mathrm{N} / \mathrm{A}$ & N/A & $\mathrm{N} / \mathrm{A}$ \\
\hline Acquisition time (min:s) & $3: 11$ & $1: 39$ & $0: 34$ & $0: 50$ & $8: 00$ \\
\hline
\end{tabular}

$b$-values of 0 and $200 \mathrm{~s} / \mathrm{mm}^{2}$, while 18 acquisitions were averaged for the $b$-value of $800 \mathrm{~s} / \mathrm{mm}^{2}$; this allowed for approximately equal signal-to-noise ratios [34] for all three $b$-values. DW-MRI was acquired over 10 sagittal slices centered on the tumor with $5 \mathrm{~mm}$ thickness and no slice gap. Spectrally selective adiabatic inversion recovery fat suppression was included for a total scan time of $1 \mathrm{~min} 39 \mathrm{~s}$.

The $T_{1}$ map and DCE-MRI scans were acquired using a three-dimensional spoiled gradient-echo sequence over 10 sagittal slices with slice thickness of $5 \mathrm{~mm}$. To construct a map of the longitudinal relaxation rate $\left(T_{1}\right)$, variable flip angle data with 10 flip angles $(2,4, \ldots, 20)$ were acquired in a total scan time of $50 \mathrm{~s}$. A Siemens TurboFLASH sequence was used to map the $B_{1}$ field to correct for transmit inhomogeneity. Due to the inclusion of a slice gap in the $B_{1}$ mapping protocol, two acquisitions were performed to cover the same field of view (FOV) as the $T_{1}$ measurements for a total acquisition time of $34 \mathrm{~s}$. DCE scans were acquired with a temporal resolution of $7.27 \mathrm{~s}$ for eight minutes. A catheter placed within an antecubital vein delivered a gadolinium-based contrast agent $(0.1 \mathrm{mmol} / \mathrm{kg}$ of Multihance or $10 \mathrm{~mL}$ of Gadovist) at $2 \mathrm{~mL} / \mathrm{s}$ followed by a $20 \mathrm{~mL}$ saline flush via a 
power injector after the acquisition of the first minute of dynamic scans which served as baseline. A separate highresolution anatomical MRI was performed before and after contrast agent administration to aid manual tumor segmentation.

\section{Tumor segmentation}

The tumor was first manually segmented on 2D slices with guidance by a certified fellowship-trained breast radiologist. This segmentation was used to calculate Response Evaluation Criteria in Solid Tumors (RECIST; [35]) measurements of longest tumor diameter. Following the manual segmentation, the tumor region of interest (ROI) was then automatically refined to detect enhancing tumor voxels using methods adapted from Giger et al. [36] and further described in our previous work [37] and Additional file 1: Methods.

\section{Image analysis}

Prior to image analysis, all images for each patient from a single scan session were aligned to a common space via rigid registration (imregtform, MATLAB) to minimize patient motion.

The DW-MRI was used to extract ADC values (see Additional file 1: Methods for details) for every voxel within the tumor and the mean ADC within the tumor was calculated. Tumor cellularity at each voxel was calculated from ADC values as previously described [38] (see Additional file 1: Methods for details). To calculate cellularity at each voxel, we assume that a tumor voxel with the minimum $\mathrm{ADC}, \mathrm{ADC}_{\text {min }}$, contains the maximum number of cells, while voxels with an ADC equivalent to free water, $\mathrm{ADC}_{\mathrm{w}}$, are devoid of tumor cells. Then, by assuming an individual tumor cell has a volume of $4189 \mu^{2}$ to derive the carrying capacity of each voxel, $\Theta$, we approximate the total number of tumor cells within each voxel by computing $\Theta *\left[\left(\mathrm{ADC}_{\mathrm{w}}-\mathrm{ADC}(x)\right) /\right.$ $\mathrm{ADC}_{\mathrm{w}}-\mathrm{ADC}_{\text {min }}$ ).

$T_{1}$ maps were generated from the variable flip angle data along with a $B_{1}$ map to correct for inaccuracies in the transmitted $B_{1}$ field as is commonly seen in gradientecho acquisitions at higher fields [39]. Details on this process are presented in Additional file 1: Methods.

The DCE-MRI data were analyzed using the Patlak model [40], which returns estimates of $K^{\text {trans }}$ using the first $36.35 \mathrm{~s}$ (i.e., the first five post-contrast injection images) of the time course. Detailed description of this analysis is provided in Additional file 1: Methods. Due to evidence of non-normality in the voxel distribution of $K^{\text {trans }}$, median tumor $K^{\text {trans }}$ values were calculated. A 'bulk tumor flow' parameter (in units of $[\mathrm{ml} / \mathrm{min}]$ ) was also calculated as the product of the mean $K^{\text {trans }}$ value of the tumor and the tumor volume.

\section{Pathological response}

Histopathologic analysis was performed at study sites as standard of care and reported to the referring oncologist. pCR was the reference standard for determining response, defined as no residual invasive disease in either breast or axillary lymph nodes after NAT. Patients were categorized as having pCR or non-pCR on the basis of postsurgical histopathologic examination findings.

\section{Statistical analysis}

Linear regression was performed to quantify changes in each MRI parameter over time across the entire cohort. Differences in baseline (MRI 1) parameters between the $\mathrm{pCR}$ and non-pCR cohorts were assessed by the Mann-Whitney test. The percent change in each imaging parameter relative to baseline was calculated by determining the relative change from MRI 1 to each subsequent MRI. Multiple Mann-Whitney tests were used to compare $\mathrm{pCR}$ versus non-pCR groups across the treatment time course. The Holm-Sidak method was used to adjust $\mathrm{p}$ values for multiple comparisons across time points. Differences between the pCR and non-pCR groups were considered significant when the adjusted $\mathrm{p}$ values were less than 0.05 . Receiver operating characteristic (ROC) curves were generated for the relative change in each MRI parameter post baseline measurement. The ability of each parameter to discriminate between the $\mathrm{pCR}$ and non-pCR groups was estimated using the area under the ROC curve with corresponding 95\% confidence interval. Statistical analysis was performed using Prism 8 software (Graphpad, San Diego, CA).

\section{Results}

Prior to the start of NAT, there were no significant differences in any quantitative MRI parameters calculated at baseline (MRI 1) between patients who achieved pCR and those who did not. Additionally, there were no significant differences in any quantitative MRI parameters across the two study sites. After commencing NAT, we observed declines in tumor volume and median $K^{\text {trans }}$ and increases in mean tumor ADC in women who ultimately achieved pCR, as shown in Fig. 2 for a representative study participant who achieved pCR. In contrast, women who did not achieve pCR did not consistently demonstrate declines in either tumor size or $K^{\text {trans }}$ nor increases in ADC, as shown for a representative study participant who had stable disease (Fig. 3).

Tumor longest diameter (Fig. 4A) and volume (Fig. 4B) tended to decline over the course of NAT across the cohort of 28 study participants $(p<0.005)$. However, women who achieved $\mathrm{pCR}$ had greater $(p<0.05)$ relative declines in tumor volume at MRI 2 relative to pre-treatment values than those who did not ultimately achieve 

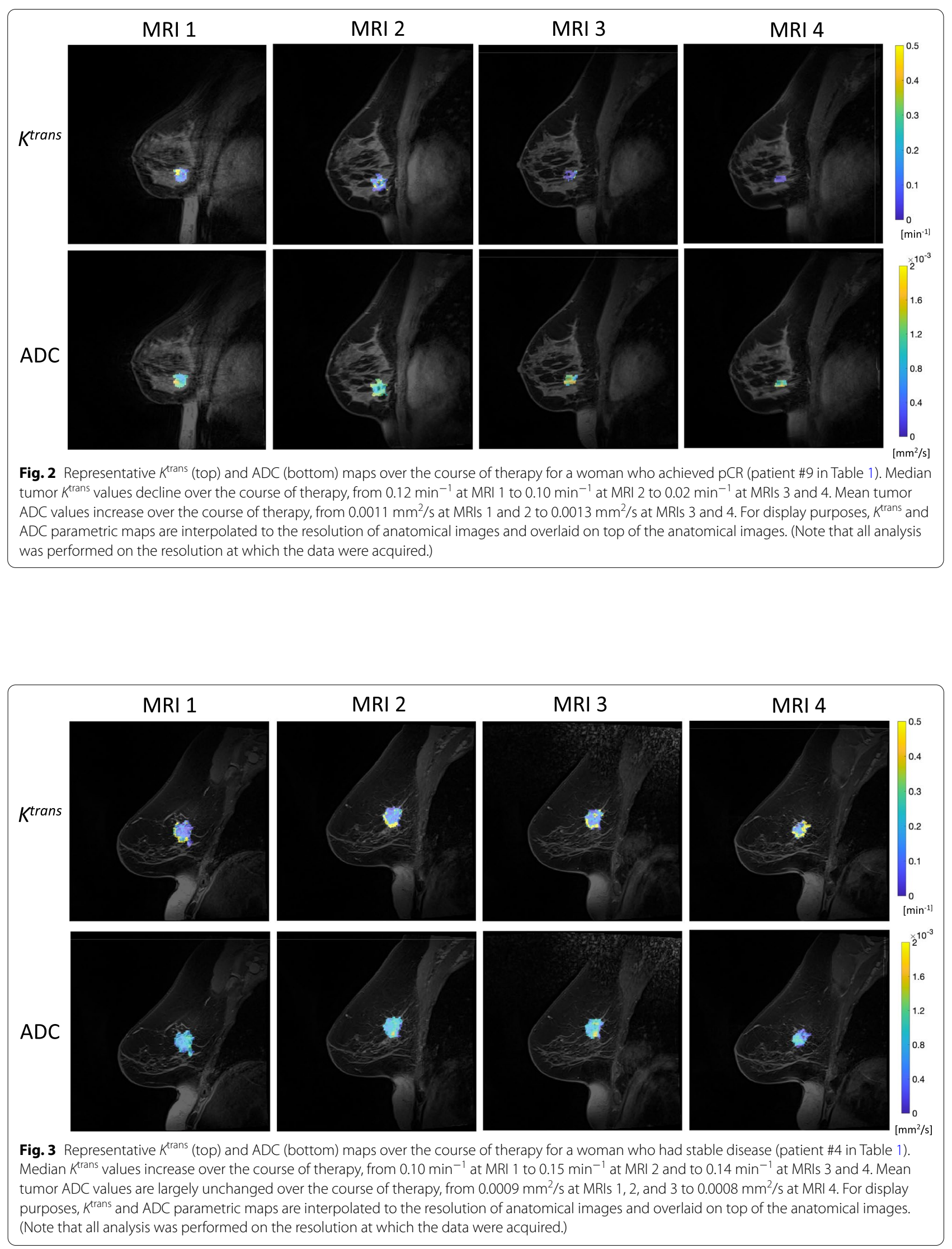
A

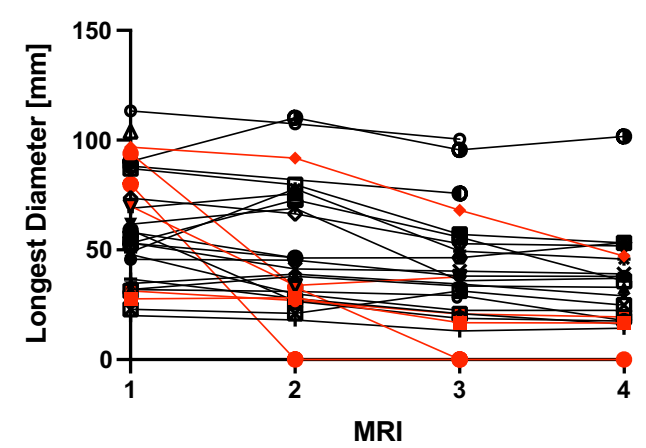

C

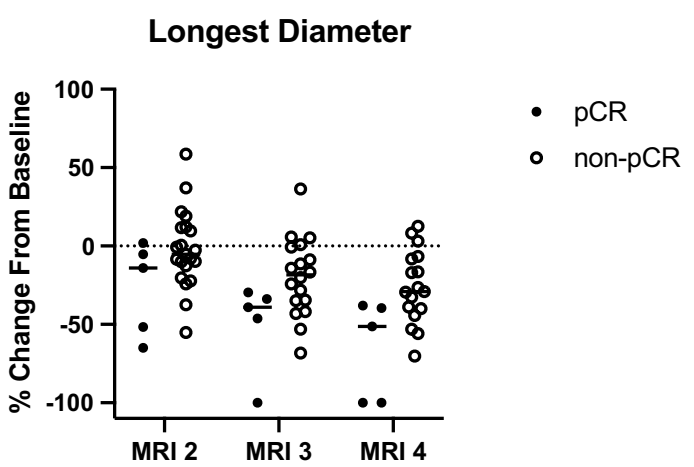

B

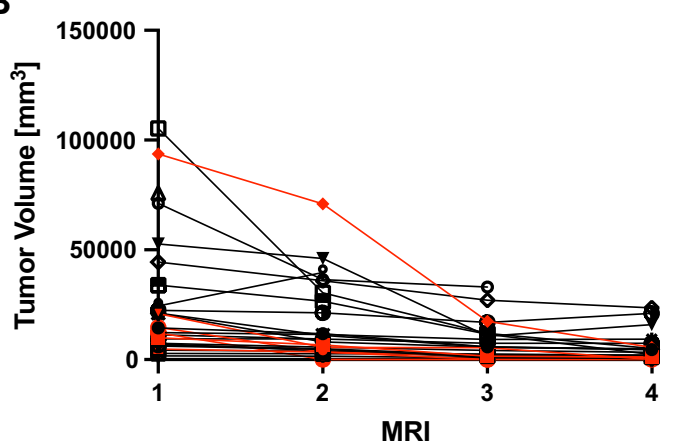

D

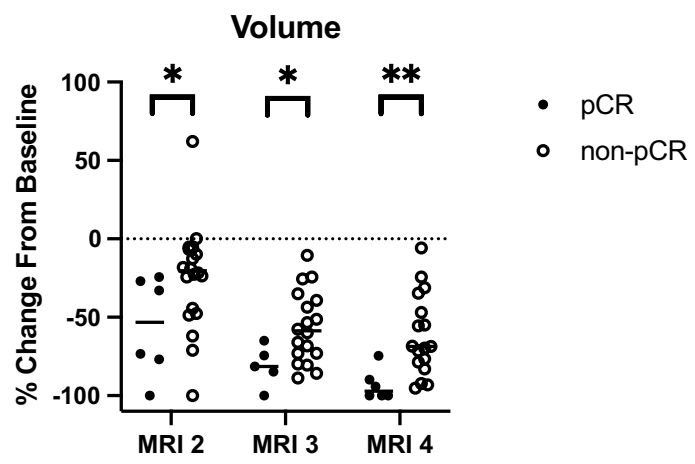

Fig. 4 Tumor longest diameter (A) and tumor volume (B) tend to decline over the course of NAT. Patients who achieved a pCR are shown in red in Panels A and B. No significant differences in the relative change in tumor longest diameter from baseline measurement at MRI 1 (C) were observed between patients who achieved pCR and those who did not achieve pCR. Significant differences in tumor volume changes from baseline (D) were observed between patients who achieved $\mathrm{pCR}$ and those who did not achieve $\mathrm{pCR}$ at all three MRIs performed during the course of therapy $\left({ }^{*} p<0.05 ;{ }^{* *} p<0.01 ;{ }^{* * *} p<0.001\right)$

pCR ( $31 \pm 15 \%$ greater decline in the pCR group). Additionally, the relative decline in tumor volume was significantly greater in the pCR group at each MRI performed during NAT $(p<0.05$, Fig. 4D). In contrast, the relative decline in tumor longest diameter was not statistically different between the pCR and non-pCR cohorts at any MRI performed during NAT (Fig. 4C).

Across the entire patient cohort, the mean tumor ADC displayed no significant change over the course of NAT (Fig. 5A). In contrast, the median $K^{\text {trans }}$ significantly declined over the course of NAT $(p<0.005$, Fig. 5B) across all patients. Changes in mean tumor ADC from baseline revealed significant differences between the pCR and non-pCR groups, with increases in the ADC in the pCR group of $21 \pm 12 \%$ and $32 \pm 14 \%$ at MRIs 3 and 4 , respectively $(p<0.05$, Fig. $5 \mathrm{C})$. Similarly, the relative decline in median tumor $K^{\text {trans }}$ was $32 \pm 15 \%$ greater in the pCR group than the non-pCR group at MRI 3 and $31 \pm 17 \%$ greater at MRI $4(p<0.05$, Fig. $5 \mathrm{D})$.

Tumor cellularity (Fig. 6A) and bulk tumor flow (Fig. 6B) tended to decline over the course of NAT across all study participants $(p<0.005)$. The decline in tumor cellularity from baseline was $30 \pm 16 \%$ greater in the pCR group at MRI 2, $24 \pm 13 \%$ greater at MRI 3 , and $33 \pm 13 \%$ greater at MRI 4 . This decline was significantly greater in the cohort who achieved pCR than the non-pCR cohort at all MRIs performed during NAT $(p<0.05$, Fig. 6C). Likewise, the relative decline in bulk tumor flow from baseline was significantly greater in the pCR group at MRIs 3 and $4(22 \pm 14 \%$ greater at MRI 3 and $19 \pm 10 \%$ greater at MRI $4, p<0.05$, Fig. $6 \mathrm{D}$ ).

The diagnostic accuracy of each MRI parameter for predicting $\mathrm{pCR}$ was quantified using $\mathrm{ROC}$ analysis. The area under each ROC curve (AUC-ROC) is shown in Table 3 with ROC curves shown in Additional file 2: Fig. S1. Change in tumor volume from baseline was the best early predictor of $\mathrm{pCR}$ in breast tumors at MRI 2 (AUC-ROC $=0.82$ ), change in mean ADC was the best predictor at MRI $3(\mathrm{AUC}-\mathrm{ROC}=0.93)$, and bulk tumor flow was the best predictor at MRI 4 (AUC$\mathrm{ROC}=1.00)$. In general, the predictive accuracy of each MRI parameter increased at later time points. 
A

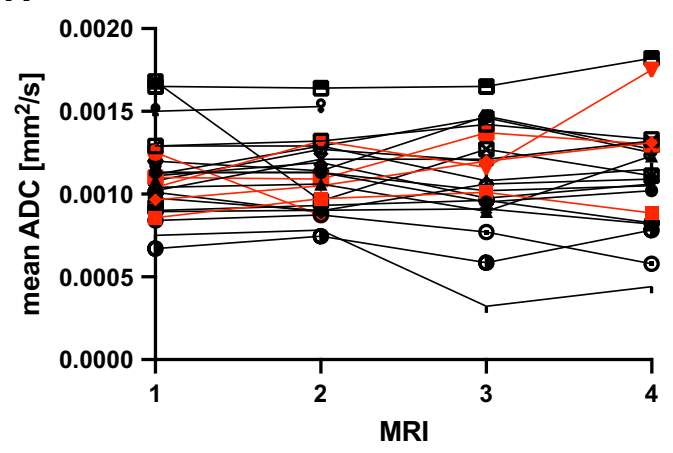

C

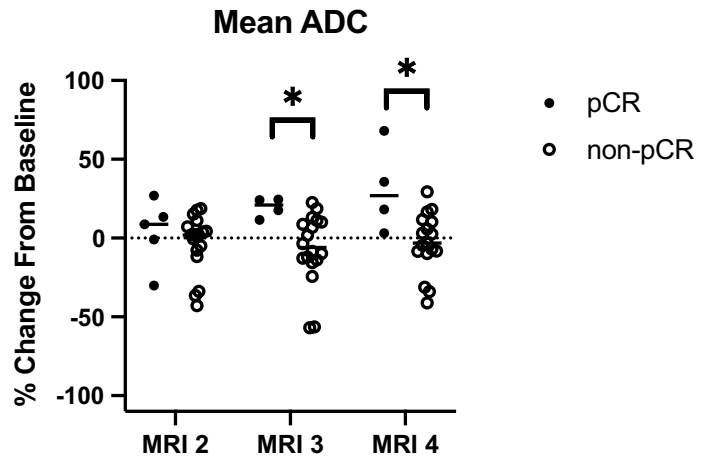

B

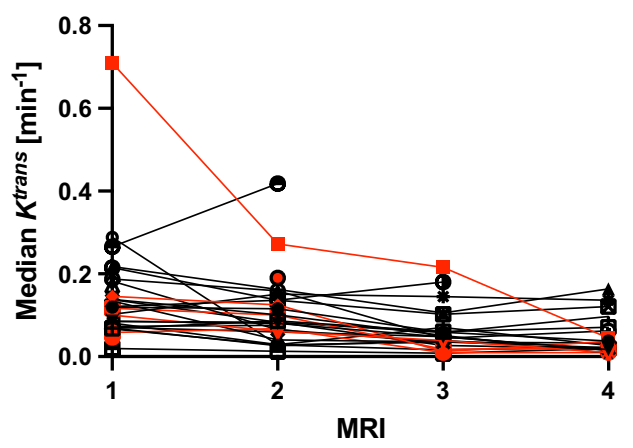

D $\quad$ Median $K^{\text {trans }}$

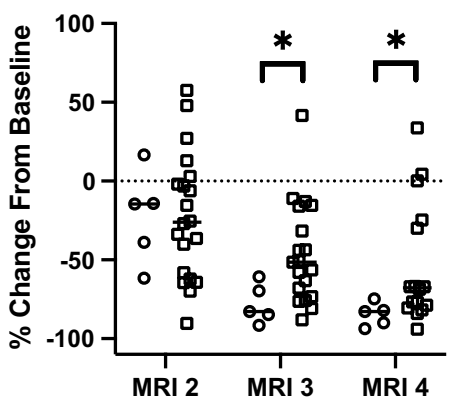

- $\mathrm{pCR}$

- non-pCR

Fig. 5 Mean tumor $\operatorname{ADC}(\mathbf{A})$ is relatively stable over the course of NAT while the median tumor $K^{\text {trans }}(\mathbf{B})$ tends to decline over the course of NAT. Patients who achieved a pCR are shown in red in Panels A and B. Significant differences in the relative change in ADC from baseline (C) were observed between patients who achieved $\mathrm{PCR}$ and those who did not achieve pCR at the third and fourth MRI. Significant differences in the relative change in $K^{\text {trans }}$ from baseline (D) were also observed between patients who achieved pCR and those who did not achieve pCR at the third and fourth MRI $\left({ }^{*} p<0.05 ;{ }^{* *} p<0.01 ;{ }^{* * *} p<0.001\right)$

\section{Discussion}

Moving advanced technology from the research-oriented, academic medical setting into the communitybased, standard-of-care setting has the potential to increase the level of care for the overwhelming majority of cancer patients. In particular, quantitative DCEand DW-MRI have emerged as two MRI techniques that have matured to the point where they can provide accurate predictions of the response of breast tumors to NAT. However, there has been limited progress implementing these quantitative MRI methods for predicting response to NAT in the community care setting. Hurdles to community implementation include technical limitations porting quantitative protocols onto MRI scanners not governed by research agreements, differences in clinical and research workflows, exam scheduling, and data transfer. We demonstrate that these challenges can be overcome. We believe this represents an important first step to disseminating quantitative imaging beyond academic and research hospitals to local and regional imaging facilities. This has the potential to greatly expand the populations of breast cancer patients that have access to (1) advanced imaging and (2) participation in clinical trials that require advanced imaging.

Previous studies have shown that DCE- and DW-MRI can predict pathological response to NAT in locally advanced breast cancer. For example, quantitative pharmacokinetic DCE-MRI performed following one cycle of NAT showed that the parameters $K^{\text {trans }}$ and $k_{e p}$ (i.e., the intravasation rate constant) were excellent predictors of pCR prior to any significant changes in RECIST [23, 29, 41]. Likewise, measurements of ADC have shown that both baseline and changes in ADC can be better predictors of tumor response than measurements of tumor size [24, 28, 42-44] and can be further strengthened by breast cancer subtype stratification [45, 46]. Combining biological information from DCE- and DW-MRI further increases the predictive ability of quantitative MRI in the neoadjuvant setting, achieving AUC-ROCs between 0.88 and $0.92[23,47]$. While these studies show positive advancements, they have yet to extend beyond the academic setting, thereby leaving an important gap in distributing this emerging technology to all patients. 
A

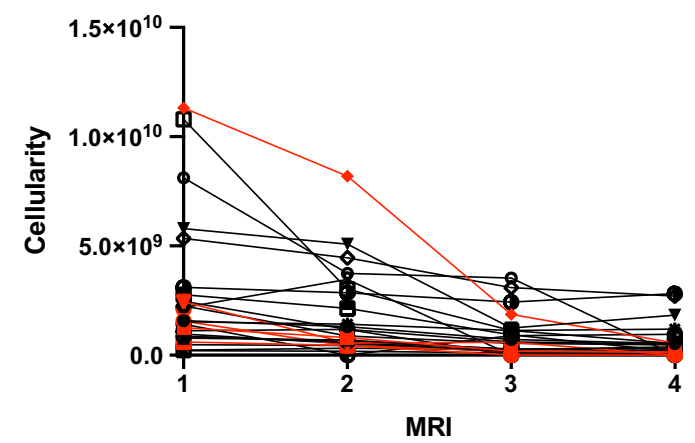

C

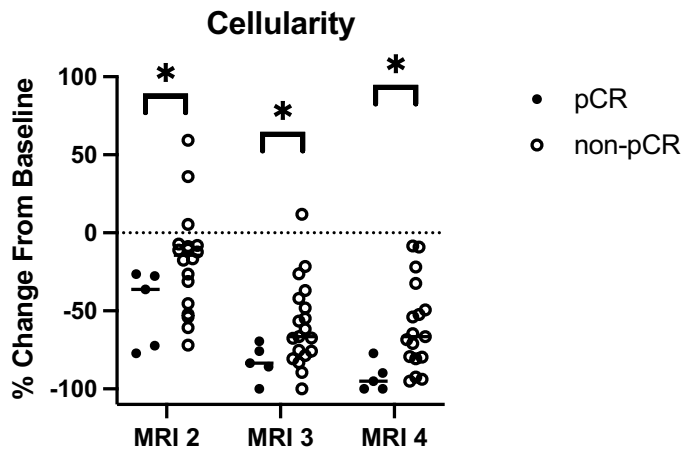

B

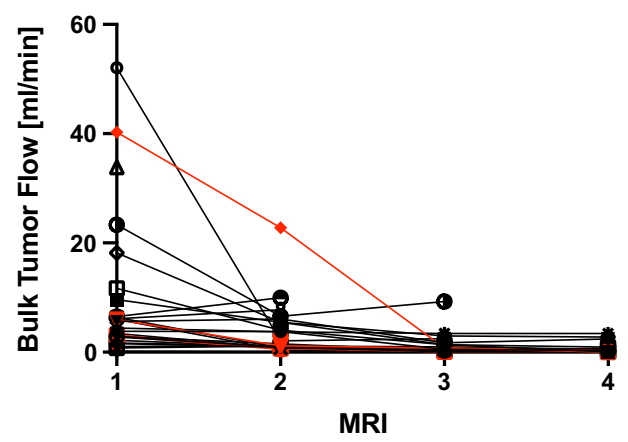

。

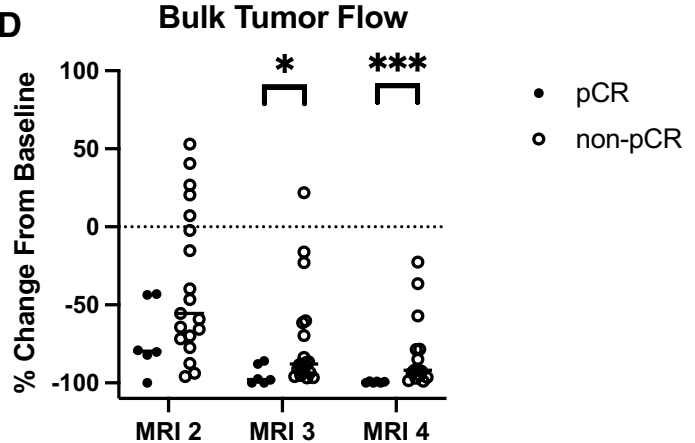

Fig. 6 Total tumor cellularity $(\mathbf{A})$ and bulk tumor flow $(\mathbf{B})$ tend to decline over the course of NAT. Patients who achieved a pCR are shown in red in $\mathbf{A}$ and $\mathbf{B}$. Significant differences in the relative change in cellularity from baseline $\mathbf{C}$ were observed between patients who achieved pCR and those who did not achieve pCR at all three MRIs following baseline. Significant differences in the relative change in bulk tumor flow from baseline $\mathbf{D}$ were observed between patients who achieved $p C R$ and those who did not achieve $p C R$ at the third and fourth MRI $\left({ }^{*} p<0.05 ;{ }^{* *} p<0.01 ;{ }^{* * *} p<0.001\right)$

Table 3 Area under the receiver operating characteristic curve (AUC-ROC) for predicting PCR from different MRI measurements

\begin{tabular}{|c|c|c|c|}
\hline & MRI 2 & MRI 3 & MRI 4 \\
\hline Longest diameter & $\begin{array}{c}0.71 \\
{[95 \% \text { Cl } 0.46-0.97]}\end{array}$ & $\begin{array}{c}0.80 \\
{[95 \% \text { Cl } 0.61-0.98]}\end{array}$ & $\begin{array}{r}0.84 \\
{[95 \% \mathrm{Cl}} \\
0.66-1.00]\end{array}$ \\
\hline Tumor volume & $\begin{array}{c}0.82 \\
{[95 \% \text { Cl } 0.65-0.99]}\end{array}$ & $\begin{array}{c}0.82 \\
{[95 \% \mathrm{Cl} 0.64-1.00]}\end{array}$ & $\begin{array}{r}0.88 \\
{[95 \% \mathrm{Cl}} \\
0.73-1.00]\end{array}$ \\
\hline Mean ADC & $\begin{array}{c}0.58 \\
{[95 \% \text { Cl 0.24-0.91] }}\end{array}$ & $\begin{array}{c}0.93 \\
{[95 \% \text { Cl } 0.81-1.00]}\end{array}$ & $\begin{array}{r}0.88 \\
{[95 \% \mathrm{Cl}} \\
0.71-1.00]\end{array}$ \\
\hline Median $K^{\text {rans }}$ & $\begin{array}{c}0.53 \\
{[95 \% \text { Cl } 0.28-0.78]}\end{array}$ & $\begin{array}{c}0.85 \\
{[95 \% \mathrm{Cl} 0.69-1.00]}\end{array}$ & $\begin{array}{r}0.84 \\
{[95 \% \mathrm{Cl}} \\
0.65-1.00]\end{array}$ \\
\hline Cellularity & $\begin{array}{c}0.81 \\
{[95 \% \text { Cl 0.62-1.00] }}\end{array}$ & $\begin{array}{c}0.83 \\
{[95 \% \text { Cl } 0.65-1.00]}\end{array}$ & $\begin{array}{r}0.88 \\
{[95 \% \mathrm{Cl}} \\
0.73-1.00]\end{array}$ \\
\hline Bulk tumor flow & $\begin{array}{c}0.73 \\
{[95 \% \text { Cl } 0.50-0.95]}\end{array}$ & $\begin{array}{c}0.81 \\
{[95 \% \text { Cl } 0.59-1.00]}\end{array}$ & $\begin{array}{l}1.00 \\
{[95 \% \mathrm{Cl}} \\
1.00-1.00]\end{array}$ \\
\hline
\end{tabular}

Each column indicates the relative change in each parameter from the baseline MRI (i.e., MRI 1) at the specified MRI scan
Our study demonstrates that the predictive ability of MRI metrics acquired in the community setting is similar to those in an academic center. In particular, we found an AUC-ROC of 0.82 after only 1 cycle of NAT using semi-automatic measurements of tumor volume. This study further introduces a novel metric we term 'bulk tumor flow' that quantitatively describes the total blood flow to the tumor. Bulk tumor flow, which combines the automated method for extracting vascularized tumor volume and the mean tumor $K^{\text {trans }}$, provided the highest overall AUC-ROC in this study, namely a perfect AUC of 1.0 at MRI 4, which was acquired at an average of 12 weeks into NAT across the cohort. Of note, the longest tumor diameter (which is used in RECIST for evaluating tumor response to therapy) was not able to predict $\mathrm{pCR}$ at any time point examined in this study. Typically, RECIST is implemented during clinical trials of highly selected patient populations receiving limited therapeutic regimens. Differences between clinical trial and 'real world' effectiveness have been well established [48]. This 'real world' effectiveness 
study of community-implemented quantitative MRI demonstrates that the MRI metrics investigated in this study may be more accurate than RECIST at predicting $\mathrm{pCR}$ across a wide range of treatments and subtypes of locally advanced breast cancer.

There are a number of limitations to this study. The same contrast agent was not used for all patients; though, each individual patient did receive the same contrast agent at all of their MRI exams. We note that this was necessary to work within multiple community imaging centers as the contrast agent and dose administered was based on site-specific protocols, and that image processing accounted for differences in contrast agent. Additionally, the treatment regimens differed between patients, as guided by the treating oncologists. While this makes the study more challenging to control for variation, it is exactly the type of patient population that community-based oncologists encounter on a daily basis and, therefore, has great practical relevance. Another area that requires further exploration for true widespread adoption is in the area of automated data analysis. We believe that the automated segmentation implemented to assess these predictions can be incorporated into routine clinical workflow; however, that has not been evaluated at this point. In this study, clinical radiologists were involved in the segmentation process, while imaging scientists conducted all quantitative analyses. An additional challenge associated with this study is the ability to conduct a protocol that works within the confines of non-research clinical scanners and within the time frame required by radiology centers performing breast MRIs. Finally, patient recruitment can be especially challenging in a non-academic environment where the oncologists, radiologists, and surgeons are not housed in one location; thus, the present study is limited to 28 patients.

\section{Conclusion}

To the best of our knowledge, this represents the first study to show that quantitative DCE- and DW-MRI can be successfully implemented in community care facilities to accurately predict the response of locally advanced breast cancer to NAT. We showed that parameters characterizing the change in tumor volume, cellularity (ADC), and vascular characteristics $\left(K^{\text {trans }}\right)$ can predict pathological complete response to NAT significantly better than the RECIST criteria. Furthermore, integrating DW-MRI and DCE-MRI with semi-automated measurements of tumor volume further increases the ability to accurately predict response early in the course of therapy. By moving these emerging measures to community-based medical centers, where the majority of patients receive their care, we are dramatically increasing the patient population that can be served by advanced imaging.

\section{Supplementary Information}

The online version contains supplementary material available at https://doi. org/10.1186/s13058-021-01489-6.

Additional file 1. Additional details describing image analysis methods. Additional file 2: Figure S1. Receiver operating characteristic curves for each MRI parameter for predicting pCR from the relative change from baseline at each serial MRI.

Acknowledgements

We sincerely thank the participation of the women who participated in this study.

\section{Authors' contributions}

JV, AGS, KPS, ASK, AMJ, JCD, SW, SA, BG, DP, and TEY performed experiments or analyzed the data. JV, AGS, and TEY wrote the manuscript. All authors reviewed and edited the final manuscript.

Funding

We thank the National Cancer Institute for support through U01CA174706, U01CA142565, U24CA226110, R01CA240589, T32EB007507, and

R01CA218700. We thank the American Cancer Society for support through RSG-18-006-01-CCE. We also acknowledge support from the Cancer Prevention and Research Institute of Texas (CPRIT) for funding through RR160005. T.E.Y. is a CPRIT scholar of cancer research.

Availability of data and materials

The imaging data used and/or analyzed in this study are available from the corresponding author upon reasonable request. All authors read and approved the final manuscript.

\section{Declarations}

Ethics approval and consent to participate

This study was approved by the University of Texas at Austin Institutional Review Board (\#2016030041).

Consent for publication

Not applicable.

Competing interests

The authors declare that they have no competing interests.

\section{Author details}

${ }^{1}$ Department of Diagnostic Medicine, University of Texas at Austin, Austin, TX 78712, USA. ${ }^{2}$ Livestrong Cancer Institutes, University of Texas at Austin, Austin, TX, USA. ${ }^{3}$ Department of Oncology, University of Texas at Austin, Austin, TX, USA. ${ }^{4}$ Oden Institute for Computational Engineering and Sciences, University of Texas at Austin, Austin, TX, USA. ${ }^{5}$ Department of Radiology, University of Alabama at Birmingham, Birmingham, AL, USA. ${ }^{6}$ Department of Biomedical Engineering, University of Alabama at Birmingham, Birmingham, AL, USA. ${ }^{7} \mathrm{O}$ Neal Comprehensive Cancer Center, University of Alabama at Birmingham, Birmingham, AL, USA. ${ }^{8}$ Department of Physics, University of Texas at Austin, Austin, TX, USA. ${ }^{9}$ Department of Radiology, University of Washington, Seattle, WA, USA. ${ }^{10}$ Austin Radiological Association, Austin, TX, USA. ${ }^{11}$ Dell Seton Medical Center at the University of Texas, Austin, USA. ${ }^{12}$ Texas Oncology, Austin, TX, USA. ${ }^{13}$ Department of Biomedical Engineering, University of Texas at Austin, Austin, TX, USA. ${ }^{14}$ Department of Imaging Physics, MD Anderson Cancer Center, Houston, TX, USA.

Received: 23 August 2021 Accepted: 17 November 2021

Published online: 27 November 2021 


\section{References}

1. Abramson RG, Arlinghaus LR, Dula AN, Quarles CC, Stokes AM, Weis JA, Whisenant JG, Chekmenev EY, Zhukov I, Williams JM, et al. MR imaging biomarkers in oncology clinical trials. Magn Reson Imaging Clin N Am. 2016:24(1):11-29.

2. Rosenkrantz AB, Mendiratta-Lala M, Bartholmai BJ, Ganeshan D, Abramson RG, Burton KR, Yu JP, Scalzetti EM, Yankeelov TE, Subramaniam RM, et al. Clinical utility of quantitative imaging. Acad Radiol. 2015:22(1):33-49.

3. Copur MS, Ramaekers R, Gonen M, Gulzow M, Hadenfeldt R, Fuller C Scott J, Einspahr S, Benzel H, Mickey M, et al. Impact of the National Cancer Institute Community Cancer centers program on clinical trial and related activities at a community cancer Center in Rural Nebraska. J Oncol Pract. 2016;12(1):67-8

4. Virostko J, Hainline A, Kang H, Arlinghaus L, Abramson R, Barnes S, Blume J, Avery S, Patt D, Goodgame B, et al. Dynamic contrast-enhanced MRI and diffusion-weighted MRI for predicting the response of locally advanced breast cancer to neoadjuvant therapy: a meta-analysis. J Med Imaging. 2018;5(1):011011.

5. Sorace AG, Harvey S, Syed A, Yankeelov TE. Imaging considerations and interprofessional opportunities in the care of breast cancer patients in the neoadjuvant setting. Semin Oncol Nurs. 2017;33(4):425-39.

6. Yankeelov TE, Abramson RG, Quarles CC. Quantitative multimodality imaging in cancer research and therapy. Nat Rev Clin Oncol. 2014;11(11):670-80.

7. Pennisi A, Kieber-Emmons T, Makhoul I, Hutchins L. Relevance of pathological complete response after neoadjuvant therapy for breast cancer. Breast Cancer (Auckl). 2016;10:103-6.

8. von Minckwitz G, Martin M. Neoadjuvant treatments for triple-negative breast cancer (TNBC). Ann Oncol. 2012;23(Suppl 6):vi35-9.

9. DeMichele A, Yee D, Berry DA, Albain KS, Benz CC, Boughey J, Buxton M, Chia SK, Chien AJ, Chui SY, et al. The neoadjuvant model is still the future for drug development in breast cancer. Clin Cancer Res. 2015;21(13):2911-5

10. Liu SV, Melstrom L, Yao K, Russell CA, Sener SF. Neoadjuvant therapy for breast cancer. J Surg Oncol. 2010;101(4):283-91.

11. Ma CX, Ellis MJ. Neoadjuvant endocrine therapy for locally advanced breast cancer. Semin Oncol. 2006;33(6):650-6.

12. Schott AF, Hayes DF. Defining the benefits of neoadjuvant chemotherapy for breast cancer. J Clin Oncol. 2012;30(15):1747-9.

13. Hayes DF, Schott AF. Neoadjuvant chemotherapy: what are the benefits for the patient and for the investigator? J Natl Cancer Inst Monogr. 2015;2015(51):36-9.

14. Bear HD, Anderson S, Brown A, Smith R, Mamounas EP, Fisher B, Margolese R, Theoret $H$, Soran A, Wickerham DL, et al. The effect on tumor response of adding sequential preoperative docetaxel to preoperative doxorubicin and cyclophosphamide: preliminary results from Nationa Surgical Adjuvant Breast and Bowel Project Protocol B-27. J Clin Oncol. 2003:21(22):4165-74

15. Carey LA, Dees EC, Sawyer L, Gatti L, Moore DT, Collichio F, Ollila DW, Sartor Cl, Graham ML, Perou CM. The triple negative paradox: primary tumor chemosensitivity of breast cancer subtypes. Clin Cancer Res. 2007:13(8):2329-34

16. Liedtke C, Mazouni C, Hess KR, Andre F, Tordai A, Mejia JA, Symmans WF, Gonzalez-Angulo AM, Hennessy B, Green M, et al. Response to neoadjuvant therapy and long-term survival in patients with triple-negative breast cancer. J Clin Oncol. 2008;26(8):1275-81.

17. von Minckwitz G. Neoadjuvant chemotherapy in breast cancer-insights from the German experience. Breast Cancer. 2012;19(4):282-8.

18. Hamy-Petit AS, Belin L, Bonsang-Kitzis H, Paquet C, Pierga JY, Lerebours F, Cottu P, Rouzier R, Savignoni A, Lae M, et al. Pathological complete response and prognosis after neoadjuvant chemotherapy for HER2positive breast cancers before and after trastuzumab era: results from a real-life cohort. Br J Cancer. 2016:114(1):44-52.

19. Abramson RG, Arlinghaus LR, Weis JA, Li X, Dula AN, Chekmenev EY, Smith SA, Miga MI, Abramson VG, Yankeelov TE. Current and emerging quantitative magnetic resonance imaging methods for assessing and predicting the response of breast cancer to neoadjuvant therapy. Breast Cancer (Dove Med Press). 2012;2012(4):139-54.

20. Hylton NM, Blume JD, Bernreuter WK, Pisano ED, Rosen MA, Morris EA, Weatherall PT, Lehman CD, Newstead GM, Polin S, et al. Locally advanced breast cancer: MR imaging for prediction of response to neoadjuvant chemotherapy-results from ACRIN 6657/I-SPY TRIAL. Radiology. 2012;263(3):663-72

21. Forte GJ, Hanley A, Hagerty K, Kurup A, Neuss MN, Mulvey TM. American Society of Clinical Oncology National Census of Oncology Practices: preliminary report. J Oncol Pract. 2013;9(1):9-19.

22. Galban CJ, Ma B, Malyarenko D, Pickles MD, Heist K, Henry NL, Schott AF, Neal CH, Hylton NM, Rehemtulla A, et al. Multi-site clinical evaluation of DW-MRI as a treatment response metric for breast cancer patients undergoing neoadjuvant chemotherapy. PLoS ONE. 2015;10(3):e0122151.

23. Li X, Abramson RG, Arlinghaus LR, Kang H, Chakravarthy AB, Abramson VG, Farley J, Mayer IA, Kelley MC, Meszoely IM, et al. Multiparametric magnetic resonance imaging for predicting pathological response after the first cycle of neoadjuvant chemotherapy in breast cancer. Invest Radiol. 2015;50(4):195-204

24. Wu LM, Hu JN, Gu HY, Hua J, Chen J, Xu JR. Can diffusion-weighted MR imaging and contrast-enhanced MR imaging precisely evaluate and predict pathological response to neoadjuvant chemotherapy in patients with breast cancer? Breast Cancer Res Treat. 2012;135(1):17-28.

25. Padhani AR, Liu G, Koh DM, Chenevert TL, Thoeny HC, Takahara T, DzikJurasz A, Ross BD, Van Cauteren M, Collins D, et al. Diffusion-weighted magnetic resonance imaging as a cancer biomarker: consensus and recommendations. Neoplasia. 2009;11(2):102-25.

26. Chenevert TL, Brunberg JA, Pipe JG. Anisotropic diffusion in human white matter: demonstration with MR techniques in vivo. Radiology. 1990;177(2):401-5.

27. Patterson DM, Padhani AR, Collins DJ. Technology insight: water diffusion MRI-a potential new biomarker of response to cancer therapy. Nat Clin Pract Oncol. 2008;5(4):220-33.

28. Sharma U, Danishad KK, Seenu V, Jagannathan NR. Longitudinal study of the assessment by MRI and diffusion-weighted imaging of tumor response in patients with locally advanced breast cancer undergoing neoadjuvant chemotherapy. NMR Biomed. 2009;22(1):104-13.

29. Tudorica A, Oh KY, Chui SY, Roy N, Troxell ML, Naik A, Kemmer KA, Chen Y, Holtorf ML, Afzal A, et al. Early prediction and evaluation of breast cancer response to neoadjuvant chemotherapy using quantitative DCE-MRI. Transl Oncol. 2016;9(1):8-17.

30. Tateishi U, Miyake M, Nagaoka T, Terauchi T, Kubota K, Kinoshita T, Daisaki H, Macapinlac HA. Neoadjuvant chemotherapy in breast cancer: prediction of pathologic response with PET/CT and dynamic contrast-enhanced MR imaging-prospective assessment. Radiology. 2012:263(1):53-63.

31. Jarrett AM, Hormuth DA 2nd, Wu C, Kazerouni AS, Ekrut DA, Virostko J, Sorace AG, DiCarlo JC, Kowalski J, Patt D, et al. Evaluating patient-specific neoadjuvant regimens for breast cancer via a mathematical model constrained by quantitative magnetic resonance imaging data. Neoplasia. 2020;22(12):820-30.

32. Weis JA, Miga MI, Arlinghaus LR, Li X, Abramson V, Chakravarthy AB, Pendyala $P$, Yankeelov TE. Predicting the response of breast cancer to neoadjuvant therapy using a mechanically coupled reaction-diffusion model. Cancer Res. 2015:75(22):4697-707.

33. Sorace AG, Wu C, Barnes SL, Jarrett AM, Avery S, Patt D, Goodgame B, Luci $J$ J, Kang H, Abramson RG, et al. Repeatability, reproducibility, and accuracy of quantitative $\mathrm{MRI}$ of the breast in the community radiology setting. J Magn Reson Imaging. 2018;48:695-707.

34. Burdette JH, Durden DD, Elster AD, Yen YF. High b-value diffusionweighted MRI of normal brain. J Comput Assist Tomogr. 2001;25(4):515-9.

35. Eisenhauer EA, Therasse P, Bogaerts J, Schwartz LH, Sargent D, Ford R, Dancey J, Arbuck S, Gwyther S, Mooney M, et al. New response evaluation criteria in solid tumours: revised RECIST guideline (version 1.1). Eur J Cancer. 2009;45(2):228-47.

36. Chen W, Giger ML, Bick U. A fuzzy c-means (FCM)-based approach for computerized segmentation of breast lesions in dynamic contrastenhanced MR images. Acad Radiol. 2006;13(1):63-72.

37. Jarrett AM, Kazerouni AS, Wu C, Virostko J, Sorace AG, DiCarlo JC, Hormuth DA 2nd, Ekrut DA, Patt D, Goodgame B, et al. Quantitative magnetic resonance imaging and tumor forecasting of breast cancer patients in the community setting. Nat Protoc. 2021;16:5309-38.

38. Atuegwu NC, Arlinghaus LR, Li X, Chakravarthy AB, Abramson VG, Sanders $M E$, Yankeelov TE. Parameterizing the logistic model of tumor growth by DW-MRI and DCE-MRI data to predict treatment response and changes in 
breast cancer cellularity during neoadjuvant chemotherapy. Transl Oncol. 2013;6(3):256-64.

39. Whisenant JG, Dortch RD, Grissom W, Kang H, Arlinghaus LR, Yankeelov TE. Bloch-Siegert B1-mapping improves accuracy and precision of longitudinal relaxation measurements in the breast at $3 \mathrm{~T}$. Tomography. 2016;2(4):250-9.

40. Karakatsanis NA, Zhou Y, Lodge MA, Casey ME, Wahl RL, Zaidi H, Rahmim A. Generalized whole-body Patlak parametric imaging for enhanced quantification in clinical PET. Phys Med Biol. 2015:60(22):8643-73.

41. Drisis S, Metens T, Ignatiadis M, Stathopoulos K, Chao SL, Lemort M. Quantitative DCE-MRI for prediction of pathological complete response following neoadjuvant treatment for locally advanced breast cancer: the impact of breast cancer subtypes on the diagnostic accuracy. Eur Radiol. 2016;26(5):1474-84

42. Shin HJ, Baek HM, Ahn JH, Baek S, Kim H, Cha JH, Kim HH. Prediction of pathologic response to neoadjuvant chemotherapy in patients with breast cancer using diffusion-weighted imaging and MRS. NMR Biomed. 2012;25(12):1349-59.

43. Park SH, Moon WK, Cho N, Song IC, Chang JM, Park IA, Han W, Noh DY. Diffusion-weighted MR imaging: pretreatment prediction of response to neoadjuvant chemotherapy in patients with breast cancer. Radiology. 2010;257(1):56-63.

44. Bufi E, Belli P, Costantini M, Cipriani A, Di Matteo M, Bonatesta A, Franceschini G, Terribile D, Mule A, Nardone L, et al. Role of the apparent diffusion coefficient in the prediction of response to neoadjuvant chemotherapy in patients with locally advanced breast cancer. Clin Breast Cancer. 2015;15(5):370-80

45. Liu S, Ren R, Chen Z, Wang Y, Fan T, Li C, Zhang P. Diffusion-weighted imaging in assessing pathological response of tumor in breast cancer subtype to neoadjuvant chemotherapy. J Magn Reson Imaging. 2015;42(3):779-87.

46. Fukuda T, Horii R, Gomi N, Miyagi Y, Takahashi S, Ito Y, Akiyama F, Ohno S, Iwase T. Accuracy of magnetic resonance imaging for predicting pathological complete response of breast cancer after neoadjuvant chemotherapy: association with breast cancer subtype. SpringerPlus. 2016;5:152.

47. Xu HD, Zhang YQ. Evaluation of the efficacy of neoadjuvant chemotherapy for breast cancer using diffusion-weighted imaging and dynamic contrast-enhanced magnetic resonance imaging. Neoplasma. 2017;64(3):430-6

48. Revicki DA, Frank L. Pharmacoeconomic evaluation in the real world. Effectiveness versus efficacy studies. Pharmacoeconomics. 1999;15(5):423-34.

\section{Publisher's Note}

Springer Nature remains neutral with regard to jurisdictional claims in published maps and institutional affiliations.
Ready to submit your research? Choose BMC and benefit from:

- fast, convenient online submission

- thorough peer review by experienced researchers in your field

- rapid publication on acceptance

- support for research data, including large and complex data types

- gold Open Access which fosters wider collaboration and increased citations

- maximum visibility for your research: over $100 \mathrm{M}$ website views per year

At BMC, research is always in progress.

Learn more biomedcentral.com/submissions 